\title{
RECEPCIÓN FÍLMICA Y NUEVAS TECNOLOGÍAS EN EL PÚBLICO DE HOY. ESTUDIO DE CASO: EL CINE ARGENTINO EN EL MARCO DE LAS PROBLEMÁTICAS DEL CINE LATINOAMERICANO.
}

\author{
Mónica Lilian Satarain 1: Universidad de Buenos Aires \\ Susana Marta Markendorf Martinez ${ }^{2}$ : Universidad de Buenos Aires
}

\section{RESUMEN}

A partir de la compleja relación del público con el cine, se intentará establecer, en el marco de la teoría de la recepción y el estudio de la opinión pública, los motivos por los que la gente ve o no cine nacional. Específicamente nos centraremos en el cine argentino en particular $y$ en el cine latinoamericano en general, intentando determinar sus formas de acceso y hábitos de consumo a estos productos culturales (salas cinematográficas, DVD tanto de edición oficial como el acceso a copias de material de libre circulación -algo muy común en toda Latinoamérica-), así como el acceso a partir del visionado en la televisión local que tiene canales dedicados a la difusión de los cines nacionales, muy especialmente de cine argentino, y la cada vez mas profusa oferta de la web, pretendemos evaluar la incidencia de las nuevas tecnologías en estos hábitos.

PALABRAS CLAVES: Recepción - cine argentino - cine latinoamericano

\section{AUDIENCE RECEPTION AND THE INFLUENCE OF NEW TECHNOLOGIES ON TODAY'S AUDIENCE. CASE STUDY: ARGENTINE CINEMA AND ITS RELATION TO THE PROBLEMS OF THE LATIN AMERICAN CINEMA.}

\section{SUMMARY}

On the basis of the complex relationship between audience and cinema, we will try to establish, taking the reception theory and the study of public opinion into consideration, the reasons why people watch, or do not watch, national cinema. We will focus on Argentine cinema in particular and Latin American cinema in general, with the aim of determining the ways in which people access and consume this cultural product (through cinemas, DVDs both of official edition and copies of materials of free circulation, which is something very common in all of Latin America). We will also study the way it is watched on local television, which has channels intended to disseminate national cinemas, mainly Argentine cinema, and the way in which it is increasingly available on the web. We will analyse the incidence of new technologies in these habits.

\footnotetext{
${ }^{1}$ Mónica Lilian Satarain: Universidad de Buenos Aires/Departamento de Artes/Bs. As./ Argentina. Correo Electrónico: monicasatarain@hotmail.com

${ }^{2}$ Susana Markendorf Martinez: Universidad de Buenos Aires/Instituto de Artes del Espectáculo/Bs As/Argentina. Correo Electrónico: smarkendorf@hotmail.com.
}

Recibido: 01/03/2010---Aceptado: 16/04/2010---Publicado: 12/11/2010 


\section{KEYWORDS: Reception - Argentine cinema - Latin American cinema}

\section{INTRODUCCIÓN}

Partiendo de la premisa de los bajos porcentajes de asistencia de público a las salas de exhibición cinematográfica con relación a los estrenos nacionales y latinoamericanos, así como la baja rentabilidad obtenida a partir de la venta y alquiler del material editado en los comercios dedicados a este rubro, la presente investigación propone indagar y problematizar el fenómeno de la recepción de estos productos de carácter simbólico. Asimismo, se intentará establecer las pautas de circulación de estos bienes culturales, teniendo en cuenta su libre difusión a través de canales alternativos como Internet, o la comercialización de copias "piratas" al alcance del público masivo a un valor ínfimo.

Las problemáticas de la recepción son un área de investigación y análisis que ha tomado un impulso fundamental en las teorías del arte en general y en las del cine en particular, en el campo de las ciencias de la comunicación y las teorías de la información, el estudio de la opinión pública como formadora del gusto. En otras disciplinas como las teorías literarias hay importantes estudios y ensayos al respecto como los de Jauss, o posteriormente Gadamer, que se han tomado como referencia para los escasos planteos que ha generado el campo específico del cine en estos casos.

La cinematografía nacional y la latinoamericana en general, sufren en la actualidad un importante desfasaje entre la aceptación internacional - el llamado boom del nuevo cine argentino - multipremiado en festivales internacionales y con muy buena distribución en algunos puntos de Europa, como España por ejemplo, sumado al incremento de los niveles de producción (los más altos desde la época de oro de los estudios, en las décadas del 30, 40 y 50 del pasado siglo) y la recepción concreta a nivel nacional y latinoamericano. Ejemplo de ello son las estadísticas presentadas anualmente en los informes elaborados por la DAC, entidad que nuclea a los Directores Argentinos Asociados. Por citar algún caso, en el año 2006, se estrenaron 242 películas de las cuales, ciento trece fueron americanas, setenta argentinas y el resto de diversos países. Sobre un total país de 34.958 .748 espectadores, (un 3\% menos que en el 2005), vieron cine argentino 4.055.764 espectadores, es decir el $11,60 \%$. En el caso de las películas argentinas, sólo siete, de las setenta estrenadas, superaron los 100.000 espectadores: "Papá se volvió loco" (1.650.000), "El aura" (600.000), "Elsa \& Fred" (540.000), "Tiempo de valientes" (470.000), "lluminados por el fuego" (330.000), "Whisky Romeo Zulu" (190.000) y "Cama adentro" (105.000). En lo que respecta al cine nacional, nos encontramos en términos porcentuales con que el diez por ciento de los estrenos convocaron al $86,33 \%$ de los espectadores del cine argentino mientras que el $90 \%$ restante atrajo al $13,33 \%$. De las setenta películas estrenadas veintisiete pertenecen al género documental; género de un alto crecimiento en los últimos años en la cinematografía latinoamericana. De estos documentales, sólo dos superaron los 10.000 espectadores. Los veinticinco restantes sólo convocaron a una muy limitada cantidad de público. La gran mayoría tuvo su estreno en las salas del circuito oficial, que tienen una cuota de pantalla muy conflictiva, resistida ampliamente por los sectores productivos del área, porque condena a los estrenos argentinos y 
latinoamericanos en general a una competencia desigual con respecto al cine extranjero, específicamente al norteamericano que cubre en red la capacidad de pantalla disponible en la región y sostiene sus estrenos con grandes inversiones publicitarias, que están vedadas por cuestiones de costos a los productores independientes.

Los espectadores, aparentemente no acompañan al cine argentino y latinoamericano en las salas de exhibición, de acuerdo a lo que sostiene la prensa especializada, así como los datos presentados, pero es llamativo que pese a las estadísticas oficiales, no existen trabajos de índole académica que respalden estas afirmaciones emanadas de fuentes oficiales y oficiosas.

La presente investigación pretende subsanar en parte este vacío, y problematizar el concepto de recepción, profundizando las razones acerca de por qué la recepción del cine argentino y latinoamericano es tan esquiva para sus propios públicos, sean éstos tradicionales o nuevos.

En el plano nacional, fuera de artículos de divulgación y estadísticas elaboradas por el INCAA y entidades vinculadas con la producción, no se encuentran otro tipo de trabajos sobre el tema. A nivel internacional, encontramos, que se ha comenzado a investigar estos temas tanto en México, país caracterizado por una alta inserción académica en el área de los estudios sobre Teorías Cinematográficas, tal el caso del trabajo realizado por la Dra Lucila Hinojosa Córdova de la Universidad Autónoma de Nuevo León, quien realizó una investigación sobre la recepción del cine mexicano en el horizonte internacional, titulado, Una Historia local en el horizonte mundial: El cine Mexicano en Monterrey; o la Dra.. María del Mar Rodríguez Rosell, de la UCAM quien desarrolla las problemáticas interculturales del cine y sus públicos en El lenguaje intercultural del Séptimo Arte. Así como en España, tal como se desprende de los trabajos realizados por Rosa María Palencia Villa de la UAB, quien ha desarrollado investigaciones sobre la recepción crítica cinematográfica y especialmente encontramos el interés de la prestigiosa revista especializada Cahiers du Cinéma publicada ahora en castellano, que dedicara un dossier en Septiembre de 2008 sobre la actualidad del cine latinoamericano, titulado Atlas de Cine Iberoamericano que desarrolla varios de los temas planteados en esta investigación. A nivel nacional, el Lic. Eduardo Schoenemann, en su trabajo titulado El nuevo cine argentino en busca de espectadores, se pregunta por el tipo de espectador que construye el llamado "nuevo cine argentino", en tanto la actual camada de realizadores tiene mayor interés en mostrar que en narrar y exhibe sin pudor las propias huellas de la enunciación, sin lograr una estructura narrativa clásica que cuenta aún con la preferencia mayoritaria del público general, que no sigue los dictados de la crítica especializada y menos de las publicaciones de origen académico.

\section{OBJETIVOS}

Nuestros objetivos de base se proponen problematizar y entender la relación existente entre los públicos de hoy con la producción fílmica y las nuevas tecnologías en el marco de una teoría de la recepción, que contemple el caso del cine argentino en el contexto de las problemáticas del cine latinoamericano. Las 
particulares coyunturas regionales y sus posibilidades de inserción real ante un público avasallado por la mundialización de la cultura instaurada desde una perspectiva global de lo económico, lo político y lo social, teniendo en cuenta que los bienes culturales relacionados con lo audiovisual, circulan por canales tradicionales y alternativos altamente influenciados por las políticas de mercado.

Para considerar las hipótesis se ha considerado por una parte que la baja circulación de estos productos por los canales oficiales se debe a problemas originados en sus mecanismos de producción, sus fallas de distribución y difusión. Por la otra se ha tomado como base del problema la modificación de hábitos y costumbres de los nuevos públicos.

Una hipótesis considera como clave el sitio de la producción, sus características propias de circulación y sus particulares dificultades de difusión, esencialmente por cuestiones económicas más que estéticas.

La otra opción focaliza el problema en el núcleo específico de la recepción: el público, signado por un lado por una fuerte tradición local (al menos en algunos países de la región). Tradición arraigada en lo latinoamericano, pero que se ve obligado a nuevos hábitos de consumo a los que irremediablemente llega arrastrado por las oleadas incesantes de mundialización de la cultura que azotan lo regional. Se busca borrar todo lo heterogéneo con el fin de lograr una homogeneización indispensable en un mercado global, o como diría el brasileño Renato Ortiz en una desterritorialización de las cosas, personas e ideas, que transforme o transfigure la realidad en virtualidades o viceversa.

Intentaremos aclarar algunos de estos interrogantes y desafíos a los cuales se enfrenta la producción simbólica en general, y muy especialmente los productos de carácter audiovisual originados en regiones no centrales.

\section{METODOLOGÍA}

Fue necesario para el cumplimiento de los objetivos planteados, hacer un trabajo de campo que contemplara tanto fuentes primarias: a través de entrevistas; observación participante y encuestas semi-estructuradas destinadas al público asistente tanto a salas de cine de exhibición tradicional, como a las dedicadas a cine arte; orientada tanto a los públicos especiales o calificados, como los asistentes a los festivales de cine. Sin desestimar el público no tradicional que sólo consume estos productos a partir de su edición en formato digital o en otros soportes, tomaremos en cuenta, además la incidencia de las nuevas tecnologías instauradas a partir del uso masivo y gratuito de la web. A tal efecto, se han preparado cuestionarios con el objeto de poder relevar las opiniones del público, a partir de una muestra del Universo. A tal efecto se realizará este relevamiento de información en diferentes zonas de Capital, Gran Buenos Aires (cines de shoppings, barriales, etc.), localidades del interior, asistencias a festivales nacionales e internacionales.

Asimismo, se trabajaron fuentes secundarias tales como estadísticas del INCAA (Instituto Nacional de Cine y Artes Audiovisuales), como de la DAC (Directores Argentinos Asociados) y otras relacionadas con la especialidad. Se contemplará 
especialmente la asistencia del público a las salas, así como las estadísticas existentes sobre la compra de material audiovisual editado, así como los canales alternativos de consumo, utilizados por usuarios habituales de programas disponibles en la red para bajar films desde diversos sitios de Internet.

Posteriormente se hará una evaluación pormenorizada de entrevistas, encuestas y estadísticas obtenidas en el transcurso de la investigación para la elaboración de un estudio crítico. El mismo deberá contemplar tanto el caudal de datos obtenidos en el transcurso de la investigación, como el rastreo de fuentes bibliográficas específicas del área de la recepción, para de esta manera lograr una articulación de corte hermeneutico sostenida tanto en los resultados concretos arrojados por esta investigación, como en la elaboración crítica desarrollada por todos los investigadores afectados a este proyecto. El equipo de investigación propuesto para este trabajo está compuesto por estudiantes del área de las artes combinadas, convocados por la dirección en la categoría de investigadores en formación, así como por docentes y graduados de las carreras de Artes (especializados en el área de Teorías Cinematográficas), así como por graduados y docentes de otras disciplinas como la Antropología Sociocultural, o la Psicología para lograr un verdadero trabajo interdisciplinario.

\section{DISCUSIÓN}

En este trabajo se presentarán las primeras conclusiones obtenidas a partir de los datos recogidos por medio de encuestas en diversos Festivales Latinoamericanos.

Al respecto cabe señalarse que se han realizado, en esta primera etapa de investigación encuestas a los asistentes a diferentes Festivales Nacionales e Internacionales de Cine, lo que significa un recorte de público minoritario que sin embargo ofrece la ventaja de un alto nivel de conocimiento específico en el tema objeto de este trabajo, como: Mar del Plata 2009; Bafici 2009 y 2010, Marfici 2009 y 2010; Morelia 2009, ( entre otros en proceso de análisis), encontrando en todos ellos un perfil de espectadores de diferentes franjas etarias, diferente formación y diferentes intereses,. Al respecto, cabe señalarse, tomándo como base 800 entrevistas realizadas, que la mayor asistencia a dichos festivales es de un público joven, la mayoría menor de 40 años (59\%) (ver cuadro 1) 


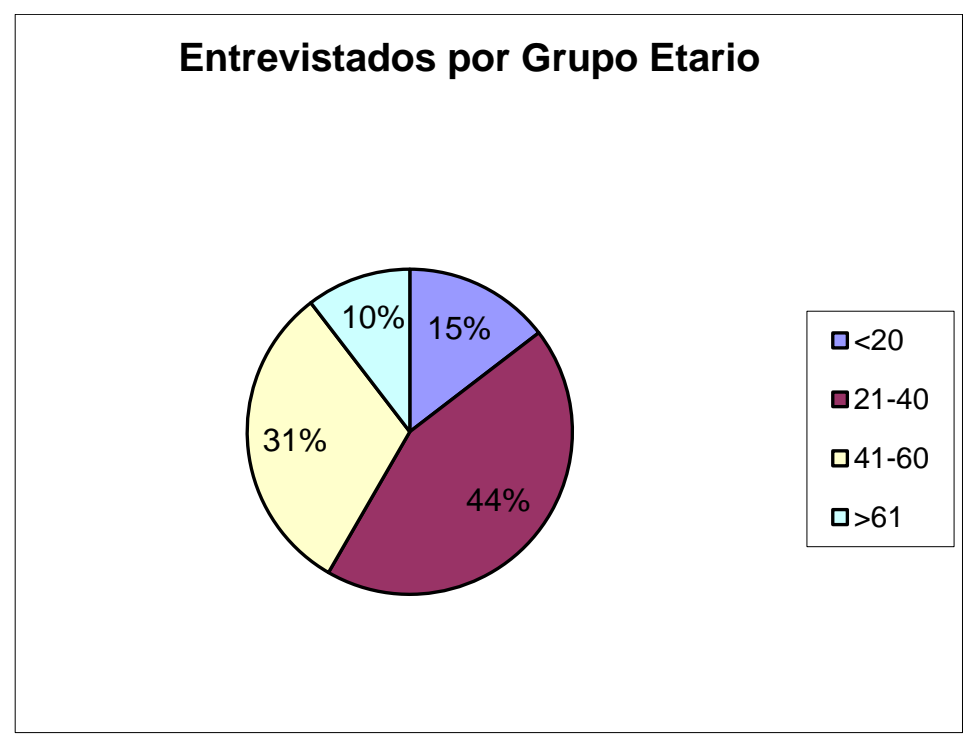

En relación a su formación nos encontramos con que el mayor porcentaje de los asistentes a estos festivales (94\%) tiene formación universitaria, y un $51 \%$ de los asistentes tienen relación con la cinematografía, ya que un $24 \%$ se presentó como periodistas o críticos de cine, y un $27 \%$ como realizadores, un $15 \%$ como docente y el $34 \%$ restante con otras profesiones, justificando su acercamiento a los festivales 0 salas cinematográficas a través de una cuestión de gusto e interés.

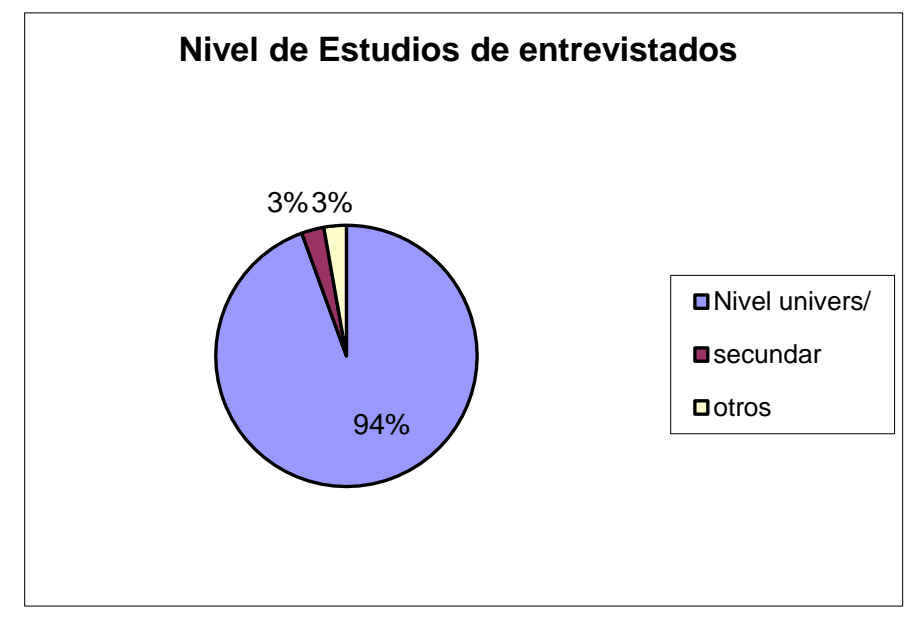

Un $10 \%$ de los asistentes a festivales reconoce que ese fue su primer festival y las expectativas de ellos coinciden en que desean ver películas que se encuentran fuera del circuito comercial. El $90 \%$ restante, coincide en esta expectativa a la que suman el ver buen cine. Casi todos ellos asistieron a ediciones anteriores de ese festival (70\%), lo que permitiría esbozar la hipótesis que los asistentes a los Festivales tienden a concurrir año tras año.

A la hora de definir el tipo de cine que les atrae, encontramos que una amplia mayoría prefiere el cine de origen Europeo, $(41 \%)$, seguido por el Latinoamericano $(29 \%)$ y el asiático (18\%). Teniendo en cuenta lo expresado sobre la preeminencia de las películas de origen norteamericano en los circuitos comerciales, es entendible la asistencia de este público a los Festivales, con el objeto de encontrar los films de su preferencia. 


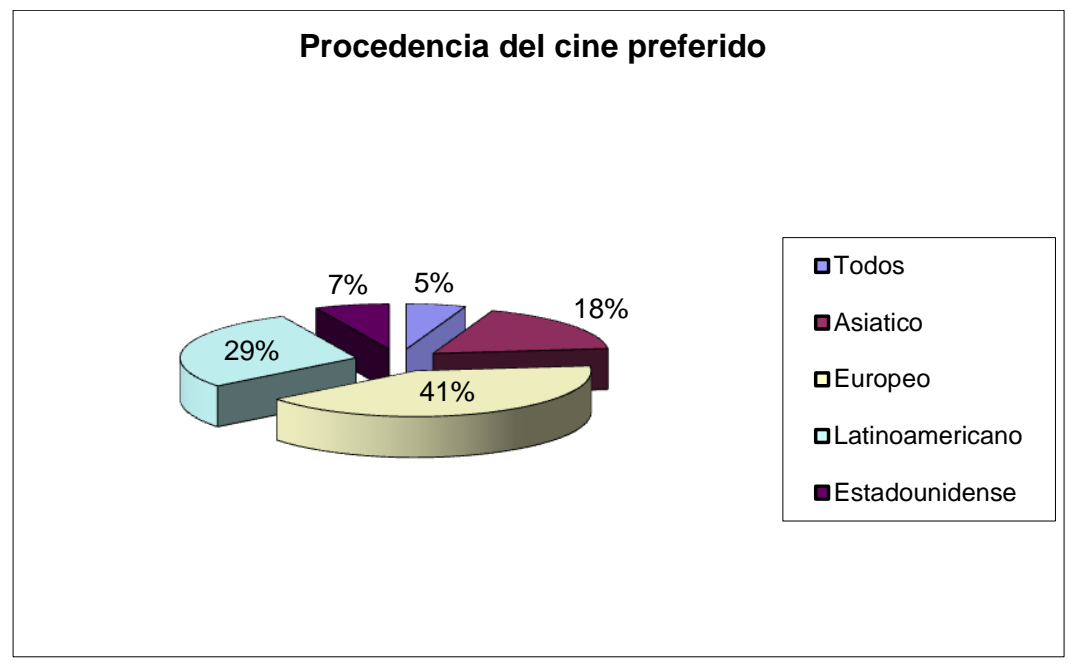

En cuanto a si prefieren cine experimental o convencional, nos encontramos con que la mayoría de los entrevistados (37\%) prefiere el cine convencional al experimental $(30 \%)$, pero un $33 \%$ no se definen, y visionan ambos tipos, reconociendo el $50 \%$ de ellos que les es indistinto en tanto el relato esté bien construido, o "esté bien hecho".

En la relación del cine Argentino con el cine Latinoamericano, un $60 \%$ de los entrevistados considera considera al primero como un representante del Cine Latinoamericano, haciendo especial hincapié en como refleja cuestiones políticas y sociales de la región tanto en los filmes de ficción como en los filmes documentales. Un 34\% que no considera esta relación,considerando en la mayoría de los casos $(70 \%)$ una mayor influencia europea, el $6 \%$ restante, relativiza su respuesta, considerando que sólo ciertos filmes reflejan el tipo de problemática que presentan los filmes latinoamericanos.

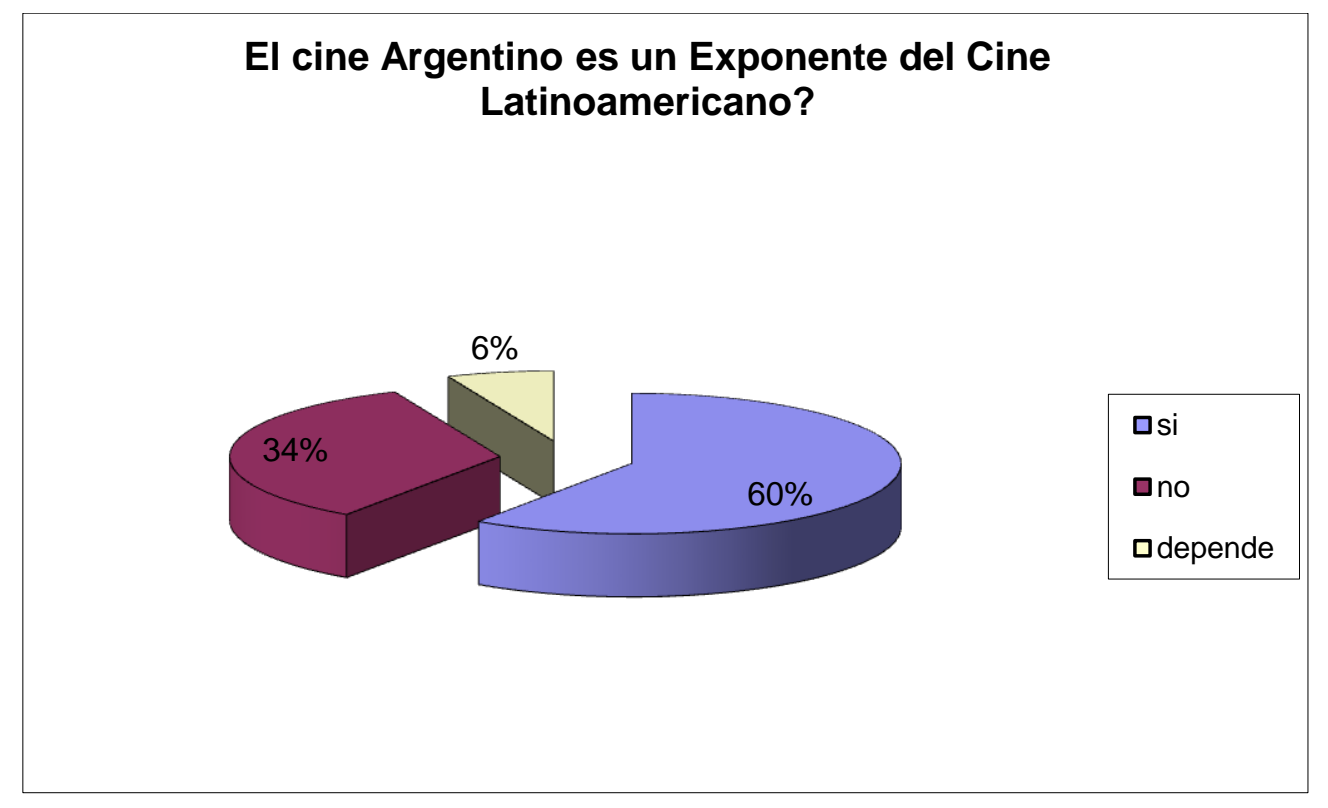


A la hora de elegir un film, el $47 \%$ de los entrevistados, sostiene que lo hace siguiendo la crítica especializada, un $30 \%$ a través del boca a boca, un $21 \%$ de acuerdo al director o a los actores (porcentaje llamativamente bajo, ya que en muchos casos el director o los actores son garantía de buen cine para muchos entrevistados,) y un $2 \%$ de acuerdo a los premios recibidos.

Todos los entrevistados reconoce ver cine argentino con frecuencia, la mayoría $(80 \%)$ mensual, un $15 \%$ reconoce que ve cine Argentino semanalmente y un $5 \%$ anualmente. Las formas de acceder a estas películas es en un $80 \%$ a través de la televisión, y en un 15\% a través de DVD. El $5 \%$ restante lo hace concurriendo al Cine, principalmente, viendo estrenos. Este porcentaje es llamativo, ya que los espacios INCAA (Cines del Instituto Nacional de Cinematografía y Artes Audiovisuales) las entradas tienen un costo menor a las de otras salas comerciales, lo que nos permitiría conjeturar que el costo de las entradas no está relacionado a una mayor concurrencia a las salas cinematográficas a ver cine nacional. En cuestión de géneros, el $60 \%$ prefiere el drama, seguido por un $30 \%$ que prefiere las comedias, un $7 \%$ los policiales y un $3 \%$ las películas "románticas"

\section{ALGUNAS CONCLUSIONES PARCIALES}

Si tenemos en cuenta que este es un trabajo en construcción, que en este momento apenas se encuentra en una etapa de recolección de datos, sólo podemos aproximar algunas conclusiones parciales y fragmentarias con respecto a los objetivos y las hipótesis de trabajo planteadas en el inicio de la investigación. Los datos recogidos en el trabajo de campo realizado en base a entrevistas y encuestas de diverso clibaje arrojan porcentajes muy parejos en las muestras recogidas en diferentes regiones ó países de Latinoamérica. Aunque parezca inverosímil en un principio, debemos rendirnos ante la evidencia concreta de que por ejemplo: el público de Michoacán (uno de los estados más importantes de México) en la histórica ciudad de Morelia -Patrimonio Cultural de la Humanidad, según la UNESCO- opina exactamente igual respecto a su cine nacional mexicano, que los asistentes a los festivales de cine de nuestra Perla del Atlántico, la turística ciudad feliz bonaerense, Mar del Plata, con respecto al cine argentino. En ambos casos las opiniones mayoritarias consideran que su cine es de escaso interés en la actualidad, que tuvo sus buenas épocas en el pasado pero que ahora carece de atractivo para el público masivo, por razones de diferente índole, las producciones nacionales son vistas por apenas unos pocos entendidos y consecuentes cinéfilos que no ahorran críticas a la hora de emitir juicios de valor sobre las mismas. Esto se repite como una constante a la hora de evaluar el cine argentino y el latinoamericano, la tendencia nos indica que hay acuerdo y similitud de opiniones, no importa si el encuestado es argentino, uruguayo, chileno, mexicano, colombiano o venezolano, si la pregunta apunta al cine nacional, las respuestas son abrumadoramente similares en cada uno de estos países. Destacamos que apartamos el caso del cine brasileño, que tiene un contexto muy particular y que no es considerado en este muestreo básicamente por razones operativas, nos circunscribimos a cine hablado en castellano para facilitar el arco de accesibilidad de los diferentes públicos nacionales, que hablan y tienen en común el mismo idioma. La pregunta a responder es qué une a todos estos públicos con sus cines, qué tipo de relaciones sociales entablan los latinoamericanos con sus productos simbólicos que los llevan a juzgarlos de tal modo. Para esto intentamos 
una aproximación interdisciplinaria que contempla tanto lo estético, como lo antropológico, lo comunicacional o lo psicoanalítico, entre otros abordajes considerados en esta investigación. Descifrar las posibles respuestas a esta pregunta se nos presenta como un acertijo asombroso, como un desafío y una especie de enigma que intuímos tiene bastante que ver con nuestras identidades como pueblos latinoamericanos y todo lo que esto significa para bien o para mal en nuestras respectivas culturas.

\section{REFERENCIAS}

\section{$\underline{\text { Libros }}$}

BAJTIN, M. (1982): Estética de la creación verbal. México, Siglo XXI.

BENJAMIN, W. (1989): Discursos interrumpidos I. Buenos Aires, Taurus.

BURCH, N. (1991): El tragaluz del infinito. Contribución a la genealogía del lenguaje cinematográfico. Madrid, Cátedra.

ECO, U. (1975): Apocalípticos e integrados ante la cultura de masas. Barcelona, Lumen.

FERRO, M. (1980): Cine e historia. Barcelona, Gustavo Gili, 1995.

GADAMER, H.G. "Verdad y Métodos: Fundamentos de una Hermenéutica Filosófica", (1977), Edic. Sigueme, España.

GARCÍA CANCLINI. N (1994) "Los Nuevos Espectadores”, Imcine.DF, México.

HALL, s. (1980): "Encoding/Decoding in television discourse", en Hall, S.;

HOBSON, D; LOWE, A.; WILLIS, P. (eds.): Culture, Media, Language. Londres, Hutchinson; 128-139.

JAUSS, H.R. "La Literatura como Provocación", (1976), Edit. Península. España.

METZ, C. (1979): El significante imaginario. Barcelona. Gustavo Gili.

MORLEY, D. (1996): Televisión, audiencias y estudios culturales. Buenos Aires, Amorrortu.

ORELL GARCIA, M (2006): "Las Fuentes del Nuevo Cine Latinoamericano", Edic. Univ. De Valparaíso, Valparaíso, Chile

OROZCO GÓMEZ, G. (1991): Recepción televisiva. México, Universidad Iberoamericana.

(2001): Televisión, audiencias y educación. Buenos Aires, Norma

(2002): "Travesías de la recepción en América Latina", en Orozco Gómez, G.

(Coord.): Recepción y mediaciones. Casos de investigación en

América Latina. Buenos Aires, Norma

ORTIZ, R. (1997): “Mundialización y cultura”. Buenos Aires, Alianza, 1997.

PICCINI, M. (1999): "Transversalidades: De las teorías de la recepción a una etnología de la cultura", en Piccini, M; Scmilchuk, G y Rosas, A.: Recepción artística y consumo cultural. México, Consejo Nacional para las Culturas y las Artes. 
RUEDA, A. (2009):"Las relaciones Norte /Sur en el Cine contemporáneo", Revista Cidob D’Afers Internacionals. № 88, Barcelona. España.

SORLIN, P. (1985): Sociología del Cine. La apertura para la historia del mañana. México, FCE.

TUDOR, A. (1975): Cine y comunicación social. Barcelona, Gustavo Gili.

$\underline{\text { Revistas }}$

AA.VV. (2008): Atlas de cine iberoamericano, Revista Cahiers du Cinéma, № 15, España.

AA.VV. (2008): Cine invisible, Revista Cahiers du Cinéma, № 14, julio, España

BAUDRY, J-L. (1970): "Cinéma: effets idéologiques produits par l'appareil de base", en Cinéthique; 7-8.

ESCALA, N. (2006):"Cine Latinoamericano en el Mercado Español". Revista latinoamericana de Comunicación, № 93, Quito, Ecuador.

$\underline{\text { Recursos electrónicos }}$

OROZCO GÓMEZ, G. (2001b): "Audiencias, televisión y educación: una deconstrucción pedagógica de la "televidencia" y sus mediaciones" en Revista Iberoamericana de Educación, 27, OEl. URL: http://www.campus-oeiorg/revista/rie27a07.htm (consultado en noviembre de 2010) 